\title{
Skeletal muscle microbiopsy: a validation study of a minimally invasive technique
}

\author{
M. Hayot*, A. Michaud", C. Koechlin*, M-A. Caron\#, P. LeBlanc", \\ C. Préfaut* and F. Maltais ${ }^{\#}$
}

ABSTRACT: The study of the peripheral skeletal muscle function in patients with chronic obstructive pulmonary disease (COPD) is of growing interest, but often requires biopsies, usually with the Bergström technique. The current study was designed to test the validity of a minimally invasive technique: the microbiopsy.

In 17 patients with COPD and four normal subjects, two specimens of the vastus lateralis were taken percutaneously under local anaesthesia, one with a 16-gauge needle (microbiopsy) and the other with the Bergström needle. The enzymatic activity of citrate synthase (CS) and phosphofructokinase (PFK), and the myosin heavy chain (MyoHC) composition were measured for both techniques.

The subjects reported no pain or much less with the microbiopsy compared with the Bergström biopsy. The microbiopsy sample weight reached $55 \pm 17 \mathrm{mg}$. The two techniques showed excellent agreement for CS activity and MyoHC composition. The PFK activity did not differ statistically between the techniques, but the agreement was moderate. The agreement between both biopsy techniques was stable over time. The median (range) fibre number within the microbiopsy specimens was 144 (38-286).

In conclusion, the current study shows the feasibility and validity of a minimally invasive muscle biopsy technique that appears more comfortable for subjects, compared with the Bergström technique.

KEYWORDS: Chronic obstructive pulmonary disease, microbiopsy, skeletal muscle

E vidence is accumulating that skeletal muscle dysfunction is frequent in patients with chronic obstructive pulmonary disease (COPD), leading to exercise intolerance, poor quality of life, and even premature mortality [1]. Research to elucidate the underlying mechanisms of muscle dysfunction has thus intensified in this field [2]. The needle biopsy technique described by BERGSTRÖM [3] is the most commonly used to assess the vastus lateralis at the cellular level, and, in fact, it has become an essential tool in biomedical research. This technique, also referred to as a semi-open biopsy, is useful for biochemical, histochemical and histomorphometric muscle analyses, with results similar to open muscle biopsy [4]. The percutaneous conchotome biopsy, also a semi-open muscle biopsy technique using an "alligator forceps", is an alternative to the Bergström technique used to biopsy muscle, such as the anterior tibialis [5]. Finally, open biopsy of the vastus lateralis [6, 7] and the deltoid muscle [8] has recently been performed in COPD patients for in vitro contractility or biochemical studies.
Although experienced groups have reported only infrequent and limited complications, these techniques may be painful for some subjects, and they need at least a $5-10-\mathrm{mm}$ skin incision. Their relative invasiveness makes it difficult to obtain several biopsies from the same subjects to study, due to, for instance, the time-course response of the skeletal muscle to interventions, such as exercise training or anabolic drug supplementation [9]. Less invasive alternatives to the semiopen muscle biopsy have been proposed by several authors working in different medical areas, such as muscular oncology [10], neuromuscular diseases $[11,12]$ and cardiac failure [13, 14]. Fine-needle muscle biopsies, also termed microbiopsies, were successfully used to obtain muscle tissue in several studies, but, to the current authors' knowledge, the results obtained with these techniques have never been compared with the semi-open techniques. In addition, the use of the microbiopsy is still limited to the few groups that have described the various techniques, and it has not gained in popularity. In the respiratory field particularly, the current authors
AFFILIATIONS

* Service Central de Physiologie Clinique, EA 701, Centre Hospitalier Universitaire Arnaud de Villeneuve, Montpellier, France.

\#Centre de Recherche, Hôpital Laval Institut Universitaire de Cardiologie et de Pneumologie, Université Laval, Québec, Canada.

CORRESPONDENCE

M. Hayot

Service Central de Physiologie Clinique

Centre Hospitalier Universitaire Arnaud de Villeneuve

Avenue du Doyen Giraud 34295 Montpellier Cedex 5

France

Fax: 33467335923

E-mail: m-hayot@chu-montpellier.fr

Received

May 052004

Accepted after revision:

November 082004

\section{SUPPORT STATEMENT}

This study was supported in part by a travelling grant from a

French-Québec cooperation programme (Ministère des Relations Internationales, QC, Canada; Ministère des Affaires Étrangères, Paris, France) 
were unable to find any study in which these techniques were used to biopsy skeletal muscles. Several potential drawbacks may be put forward to explain this: the limited weight of the samples, the questionable quality of the specimens, and uncertainty about the validity of the microbiopsy compared with the Bergström technique. Thus, the present study was designed to test the validity of the muscle microbiopsy compared with the Bergström technique, which is considered as the gold standard. Two percutaneous biopsy specimens of the vastus lateralis were obtained from the same subject, one using a 16-gauge disposable needle (microbiopsy) and the other using a Bergström needle. Muscle enzymatic activity and the distribution of the different myosin heavy chains (MyoHCs) were compared between the two techniques.

\section{MATERIALS AND METHODS Study design}

The current authors took advantage of the participation of patients with COPD and healthy subjects, who were about to undergo a needle biopsy of the quadriceps for research purposes, in order to evaluate the agreement between the microbiopsy procedure and the Bergström needle biopsy. All subjects were evaluated at the Laval Hospital exercise physiology laboratory (Québec, Canada). In a single laboratory visit, each subject underwent two consecutive percutaneous biopsies from the same site at the mid-thigh level of the vastus lateralis muscle. All biopsies were performed by the same two investigators. Under local anaesthesia, the microbiopsy was performed first with a 16-gauge disposable needle and was then followed by a percutaneous muscle biopsy, as described by BERGSTRÖM [3], with 6-mm cutting trocars. The study was approved by the institutional ethics committee, and each subject received a thorough explanation of the rationale and procedures of the present protocol and gave their informed consent.

This study was conducted over a 1-yr period, during which the subjects were recruited in three successive phases. The first phase allowed the current authors to evaluate the feasibility and safety of the microbiopsy procedure, and to optimise tissue preparation and analysis for the subsequent phases of the study. Thus, this study design also allowed the provision of useful information concerning the learning process for the microbiopsy technique.

After preliminary attempts had shown that an 18-gauge Magnum ${ }^{\circledR}$ needle was too small to provide enough muscle tissue, the first phase of the study was undertaken in five subjects using a 16-gauge Magnum ${ }^{\circledR}$ needle (1.65-mm diameter, Magnum ${ }^{\circledR}$ Needle, MN1610; Bard, Covington, GA, USA). The results in these five subjects were more satisfying: the clinical tolerance was excellent, and the total weight of 1-3 microbiopsy samples ranged from 11-20 mg. For the second and third phases of the study, nine and eight subjects were recruited, respectively. It was decided, based on the results of the first phase, to increase the number of muscle samples obtained with the microbiopsy procedure from three to six. This was done in order to increase the amount of muscle tissue obtained from the microbiopsy. Only the biological data obtained during the second and third phases were used for data analysis.
A total of 11 (eight COPD and three healthy subjects) of the 17 subjects contributed twice to the study (they had two biopsies of each type on two separate occasions). This was done to fulfil the requirements of the research protocol in which they were participating. It was not possible to directly compare the results of the first and second microbiopsies, since they were obtained in different conditions (e.g. in the contralateral legs, after muscle-fatiguing exercise). However, this did allow the current authors to study whether the agreement between the microbiopsy and the Bergström biopsy persisted over time and/or in a different condition.

\section{Study subjects}

In total, 17 male patients with COPD (mean \pm SD age $69 \pm 5$ yrs; forced expiratory volume in one second $34 \pm 12 \%$ predicted; body mass index (BMI) $24.8 \pm 3.3 \mathrm{~kg} \cdot \mathrm{m}^{-2}$ ) and four healthy male nonsmokers (age range 27-29 yrs; BMI range 20.8$23.7 \mathrm{~kg} \cdot \mathrm{m}^{-2}$ ) were studied. The diagnosis of COPD was based on previous or current smoking history and pulmonary function tests showing not fully reversible bronchial obstruction [15]. All patients were clinically stable at the time of the study. No subject had contraindications for the biopsy procedure, such as anticoagulation or known blood discrasia.

\section{Biopsy procedures}

Microbiopsy device and needles

The microbiopsy was performed with a spring-loaded and reusable instrument, the Magnum ${ }^{\circledR}$ Biopsy System (MG1522; Bard). This device is a recent version of an automated biopsy system, which has proven effective for fine-needle percutaneous biopsies of various tissues, such as liver, kidney, pancreas, prostate, uterus, and psoas muscle [16], and for the diagnosis of musculoskeletal sarcomas [10]. The current authors also used 16-gauge core disposable biopsy needles (Magnum ${ }^{\circledR}$ Needle, MN1610; Bard; length of the sampling notch $19 \mathrm{~mm}$; course $22 \mathrm{~mm}$ ) and an insertion cannula (a 14gauge i.v. catheter), which allowed multiple biopsies to be obtained through a single skin puncture.

\section{Microbiopsy procedure}

Figure 1 illustrates the successive steps of the microbiopsy procedure. After local anaesthesia with $2 \mathrm{~mL}$ of lidocaine $2 \%$ (lignocaine; fig. 1a) and placement of the biopsy needle into the biopsy device (fig. 1b), the skin was punctured with the insertion cannula perpendicular to the muscle until the fascia was pierced (fig. 1c). The biopsy needle was then inserted through the cannula after removal of its trocars (fig. 1d). A muscle sample was obtained by the activation of a trigger button, which unloaded the spring and activated the needle to collect a muscle piece. The biopsy needle was then slid out of the insertion cannula while the latter was maintained in place, thus avoiding repeated skin punctures. The muscle specimen (fig. 1e) was removed from the biopsy needle using a sterile scalpel. The sample was subsequently placed on a gauze pad, rapidly weighed (fig. 1f) and immediately frozen in liquid nitrogen and stored at $-80^{\circ} \mathrm{C}$ for further analyses. Some of the microbiopsy samples were plunged in pre-cooled isopentane for further transversal cryosection and staining. This whole biopsy procedure was repeated three (phase 1) to six (phases 2 and 3) times in order to obtain sufficient muscle tissue. The sum of the weights of the 3-6 biopsy specimens obtained 

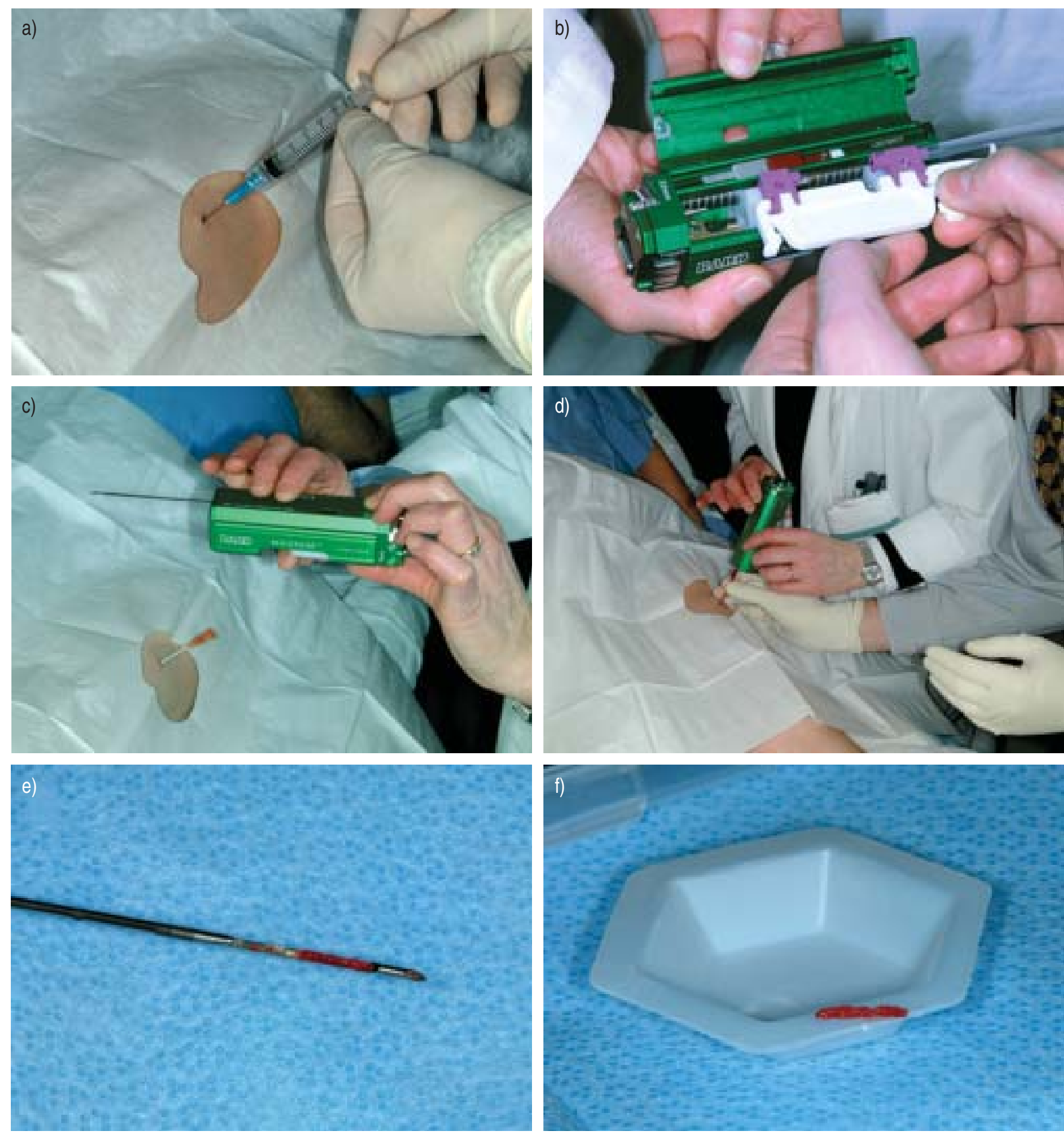

FIGURE 1. Microbiopsy procedure showing the Magnum ${ }^{\circledR}$ Biopsy System (Bard, Covington, GA, USA) device, and the successive steps of the microbiopsy, as follows: a) local anaesthesia of the area; b) placement of the biopsy needle into the biopsy device; c) skin puncture using the insertion cannula; d) insertion of the biopsy needle; e) removal of the muscle specimen; and f) weighing of the sample.

during the entire microbiopsy procedure defined the term "microbiopsy sample weight".

\section{Bergström needle biopsy}

The second biopsy was performed immediately after the microbiopsy, according to the technique described by
BERGSTRÖM [3], and without the need for further anaesthesia. Briefly, after a $1-\mathrm{cm}$ skin incision was made at the puncture site of the microbiopsy, muscle samples were obtained using one or two passes with the Bergström needle. These were immediately frozen in liquid nitrogen and stored at $-80^{\circ} \mathrm{C}$. 


\section{Clinical tolerance}

After each biopsy procedure, the subjects were asked to evaluate the associated pain using a modified Borg 0-10 scale: 0 indicated no pain at all and 10 indicated unbearable pain [17].

\section{Muscle analyses}

Muscle enzymatic activity

The muscle biopsy specimens were coded and analysed without knowledge of the clinical data. The enzyme activities from $\sim 5-10-\mathrm{mg}$ samples (same amount of muscle for the microbiopsy and the Bergström biopsies) were measured at $25^{\circ} \mathrm{C}$ and expressed in $\mu \mathrm{mol} \cdot \mathrm{g}^{-1} \cdot \mathrm{min}^{-1}$ wet weight of muscle. The activities of citrate synthase (CS; enzyme classification (EC) 4.1.3.7) and phosphofructokinase (PFK; EC 2.7.1.11) were measured using a spectrophotometric technique as previously described [18, 19]. The reaction media used to measure the enzymatic activities were tris- $\mathrm{HCl} 150 \mathrm{mM}(\mathrm{pH}$ 8.0) containing 5,5'-dithio-bis(2-nitrobenzoic acid) $0.16 \mathrm{mM}$, acetyl-CoA $1.235 \mathrm{mM}$ and sodium oxaloacetate $0.38 \mathrm{mM}$ for CS, and tris$\mathrm{HCl} 50 \mathrm{mM}$ (pH 8.2) containing $\mathrm{MgCl}_{2} 6 \mathrm{mM}, \mathrm{KCl} 250 \mathrm{mM}$, ATP $1 \mathrm{mM}$, AMP $0.17 \mathrm{mM}$, nicotinamide adenine dinucleotide (reduced form) $0.17 \mathrm{mM}, \mathrm{KCN} 1 \mathrm{mM}$, fructose-6phosphate $3 \mathrm{mM}$, glycerol-3-phosphate dehydrogenase $10 \mathrm{U}$, aldolase $0.9 \mathrm{U}$ and triose-phosphate isomerase 9.6 U for PFK. Each assay was performed in duplicate and the average of the two values is reported.

\section{Skeletal muscle myosin heavy chain isoform expression Sample preparation for sodium dodecylsulphate-poly-acrylamide gel electrophoresis}

Muscle biopsy samples ( 5-10 mg) were homogenised on ice with a tissue grinder, in a buffer solution (sucrose $210 \mathrm{mM}$, HEPES $30 \mathrm{mM}$, EGTA $2 \mathrm{mM}, \mathrm{NaCl} 40 \mathrm{mM}$ and EDTA $5 \mathrm{mM}$, $\mathrm{pH}$ 7.4) containing a $1 \%$ protease inhibitor cocktail (Sigma, Oakville, Canada). Samples were centrifuged, and pellets were resuspended in a second buffer containing $\mathrm{KCl} 175 \mathrm{mM}$, EDTA $5 \mathrm{mM}$, Tris $20 \mathrm{mM}$ and Triton X-100 0.5\%, pH 6.8, to solubilise the membrane-bound components. The remaining pellets were then resuspended twice in a third buffer containing $\mathrm{KCl}$ $150 \mathrm{mM}$, EDTA $5 \mathrm{mM}$ and Tris $20 \mathrm{mM}$, pH 7.0. Protein concentration was measured according to the method of BRADFORD [20]. Aliquots of myofibrils were dissolved in a sodium dodecylsulphate gel-loading buffer and frozen at $-80^{\circ} \mathrm{C}$.

\section{Myosin heavy chain isoform resolution}

MyoHC was resolved on $8 \%$ polyacrylamide gels containing $30 \%$ glycerol. Following electrophoresis, the gels were stained with Coomassie blue. MyoHC isoforms I, IIa and IIx were identified by order of migration, according to ENNION et al. [21] and SMERDU et al. [22]. The gels were scanned electronically and the relative proportions of $\mathrm{MyoHC}$ were measured using Scion Image Beta 4.02 (Scion Corp., Frederick, MD, USA). The results are expressed as the percentage of total $\mathrm{MyoHC}$ in each sample.

\section{Muscle sample staining}

An attempt was made to determine the number of fibres in the microbiopsy specimens, although this was not the main objective of the current study. When sufficient tissue was available, a portion of the biopsy specimens was preserved for further cryosection. In these cases, a muscle sample removed from the needle with a scalpel was rapidly embedded in Optimum Cutting Temperature ${ }^{\circledR}$ (Sakura Finetek, Torrance, CA, USA), then plunged into pre-cooled isopentane and stored at $-80^{\circ} \mathrm{C}$. Transverse cryosections at $-20^{\circ} \mathrm{C}(10-\mu \mathrm{m}$ thick) were subsequently made and stained with either haematoxylin and eosin or myosin ATPase. The sections were observed under light microscopy $(200 \times$ magnification), and the whole surface was photographed with a digital camera and reconstituted when needed. The number of fibres was then counted using a personal computer.

\section{Statistics}

The values are expressed as mean $\pm \mathrm{SD}$. The $\mathrm{t}$-test for paired observations was used for mean comparisons of the microbiopsy and Bergström sample analyses. Values of $\mathrm{p}<0.05$ were considered significant. As proposed by BLAND and ALTMAN [23], comparisons between the microbiopsy and Bergström sample analyses were performed by means of the limits of agreement, where individual differences (Bergström minus microbiopsy data) were plotted against the corresponding mean values. From these data, limits of agreement were then calculated (i.e. mean difference between the Bergström and microbiopsy methods \pm 1.96 SD) [23].

\section{RESULTS \\ Clinical tolerance}

There was little or no pain associated with the microbiopsy, with a pain score ranging $0-1$, while it ranged 4-6 with the Bergström biopsy. The patients were also asked the following question: "If another muscle biopsy were necessary, which one (microbiopsy or Bergström biopsy) would you prefer?" All subjects chose the microbiopsy.

\section{Microbiopsy sample weight}

The mean \pm SD microbiopsy sample weight increased from $15 \pm 3 \mathrm{mg}$ for the first phase to $27 \pm 9 \mathrm{mg}$ (range 9-45 mg) and $55 \pm 17 \mathrm{mg}$ (range $32-90 \mathrm{mg}$ ) for the second and third phases, respectively. The total sample weights from the Bergström biopsy were $218 \pm 75 \mathrm{mg}, 145 \pm 64$ and $217 \pm 89$ for the three phases of the study, respectively. There was no significant relationship between the BMI of the COPD patients, which may reflect muscle wasting, and the microbiopsy sample weights.

\section{Agreement study}

In order to perform all three validation measurements (CS, $\mathrm{PFK}$ and $\mathrm{MyoHC}$ isoform expression), $30 \mathrm{mg}$ of muscle were necessary. This amount of muscle tissue could be obtained with the microbiopsy procedure in 11 out of 17 subjects of phases 2 and 3. Therefore, due to the reduction in tissue amount with the dosage process, the agreement between the two biopsy procedures could be evaluated in 17 patients for CS, but only in 14 and eight subjects for PFK and MyoHC isoform expression, respectively.

\section{Agreement for citrate synthase activity}

Figure $2 \mathrm{a}$ shows that the mean values of the two biopsy techniques did not statistically differ. Figure $2 b$ shows the individual differences between the two techniques plotted 

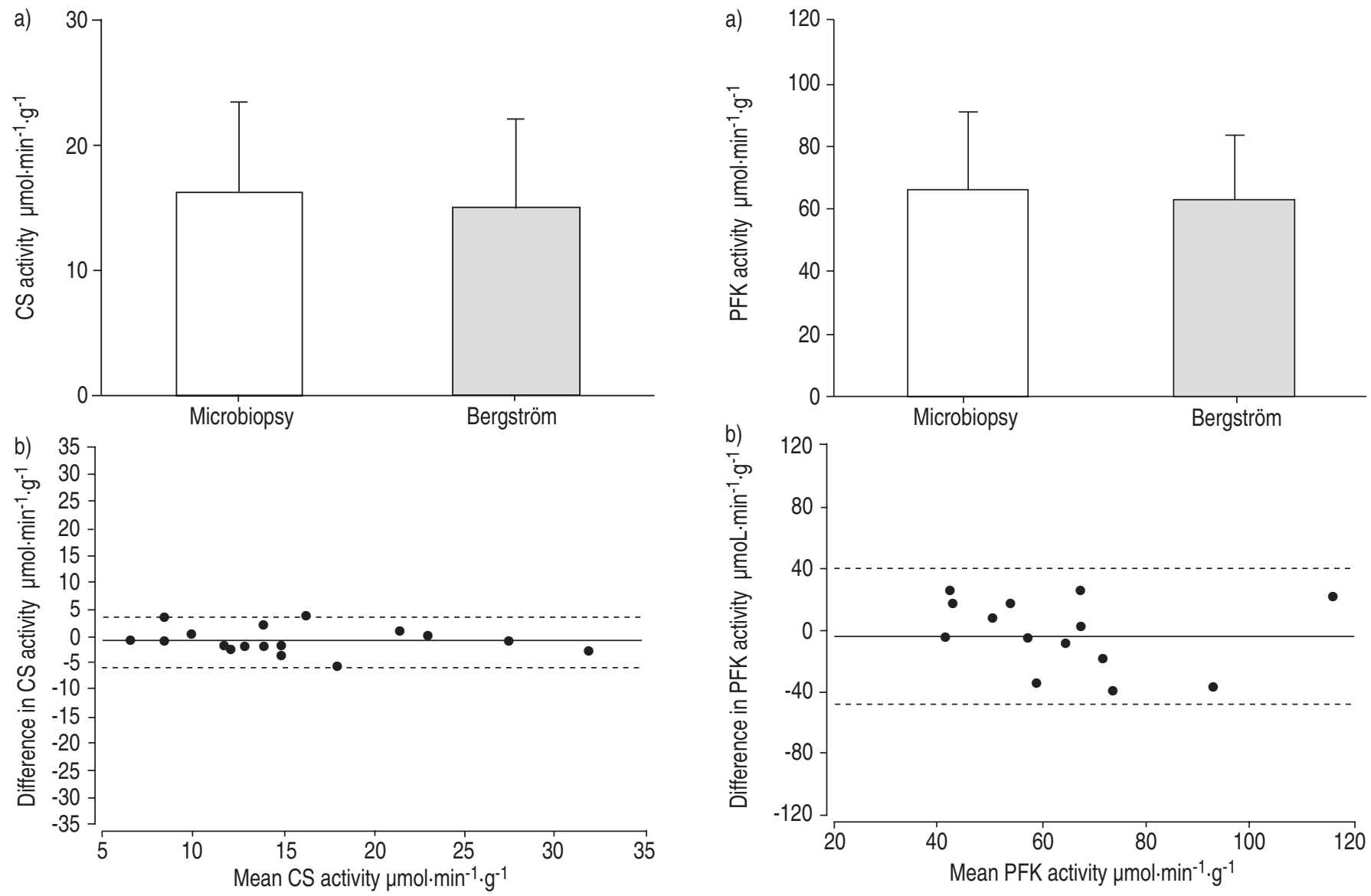

FIGURE 2. Citrate synthase (CS) activity in the microbiopsy and Bergström biopsy samples. a) Comparison of mean \pm SD values between the microbiopsy and Bergström biopsy samples. b) Bland and Altman graphical representation of CS activity obtained with the two techniques during the first biopsy in 17 subjects ( $\square$ : mean; -----: $\pm 1.96 \mathrm{SD})$.

against the corresponding mean values, according to the Bland and Altman method. The mean difference was low $\left(-0.99 \mu \mathrm{mol} \cdot \mathrm{min}^{-1} \cdot \mathrm{g}^{-1}\right)$, and the limits of agreement were tight, ranging $3.77--5.75 \mu \mathrm{mol} \cdot \mathrm{min}^{-1} \cdot \mathrm{g}^{-1}$, which was close to the duplicate results. This figure illustrates the excellent agreement between these two biopsy techniques. No relationship was found between the BMI of the COPD patients and the difference in CS activity between the two biopsy techniques. In other words, a low BMI did not cause a discrepancy between the two techniques.

\section{Agreement for phosphofructokinase activity}

Comparisons for the PFK enzymatic activity between the microbiopsy and the Bergström technique are provided in figure 3. There was no significant difference between the two techniques (fig. 3a). Figure $3 \mathrm{~b}$ shows the individual differences between the two techniques plotted against the corresponding mean values. The mean difference between the two techniques during the first biopsy was small $\left(-3.7 \mu \mathrm{mol} \cdot \mathrm{min}^{-1} \cdot \mathrm{g}^{-1}\right)$, but the limits of agreement were wide (ranging 40.0- $-47.4 \mu \mathrm{mol} \cdot \mathrm{min}^{-1} \cdot \mathrm{g}^{-1}$ ), indicating moderate agreement. Again, no relationship was found between BMI

FIGURE 3. Phosphofructokinase (PFK) activity in the microbiopsy and Bergström biopsy samples. a) Comparison of mean \pm SD values between the microbiopsy and Bergström biopsy samples. b) Bland and Altman graphical representation of PFK activity obtained with the two techniques during the first biopsy in 14 subjects ( - : mean; ----: $\pm 1.96 \mathrm{sD}$ ).

and the difference in PFK activity between the two biopsy techniques in the patients with COPD.

\section{Myosin heavy chain electrophoresis}

Figure 4a shows the different migration rates of MyoHC types I, IIa and IIx in both the microbiopsy and the Bergström samples of two representative subjects. This figure also compares the MyoHC expression between the two techniques. There was no statistically significant difference in the relative contents of MyoHC types I, IIa and IIx between the two techniques (fig. 4b). Figure 4c shows the Bland and Altman graphical representation when the different MyoHC types were pooled, with a mean difference of $0.02 \%$ and limits of agreement of $15.65--15.62 \%$, which can be considered as excellent.

\section{Agreement between the two biopsy techniques when repeated over time}

A second pair of measurements for CS, PFK and MyoHC could finally be obtained in 10, four and four subjects, respectively, in whom two pairs of biopsies (two microbiopsies and two Bergström biopsies) were obtained. Figure 5 illustrates the 

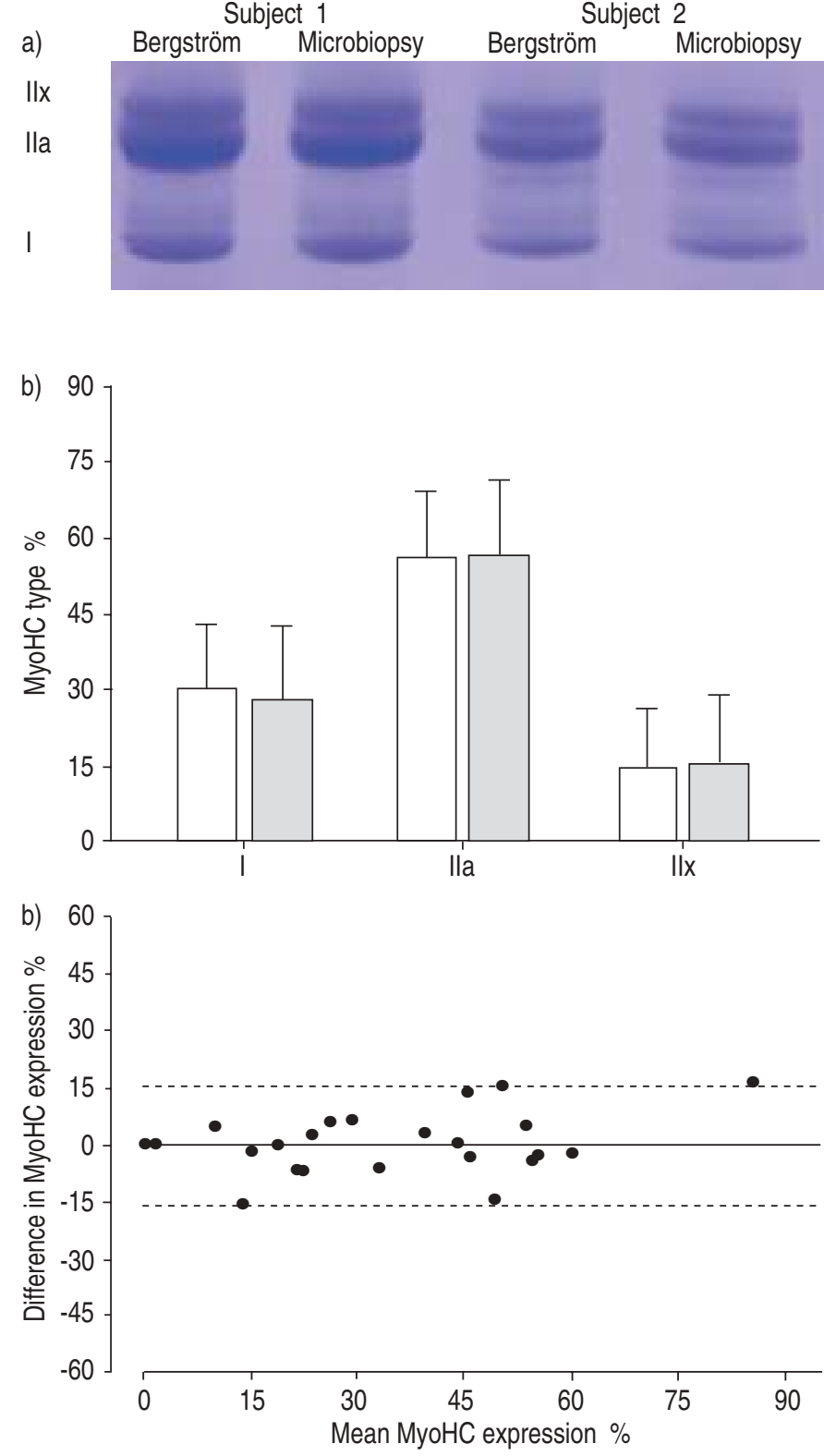

FIGURE 4. Myosin heavy chain (MyoHC) expression. a) Different migration rates of MyoHC types I, Ila and IIx in the microbiopsy and the Bergström samples of two representative subjects. b) Comparison of MyoHC types I, Ila and IIx expression between the microbiopsy $(\square)$ and the Bergström technique ( $\square$ ). c) Bland and Altman graphical representation of the different $\mathrm{MyOHC}$ isoforms obtained with the two biopsy techniques (-: mean; -----: $\pm 1.96 \mathrm{SD}$ )

agreement between the two biopsy techniques in these subjects. As can be seen, the second biopsy data were similarly distributed around the mean difference of the initial group and were within their limits of agreement. This indicates that the microbiopsy provides a similar level of agreement as the Bergström biopsy when repeated biopsies are made in the same subject over time and in another condition.

\section{Microscopic observation of muscle tissue}

The tissue quality was initially poor and/or only a low number of fibres could be counted. This progressively improved over
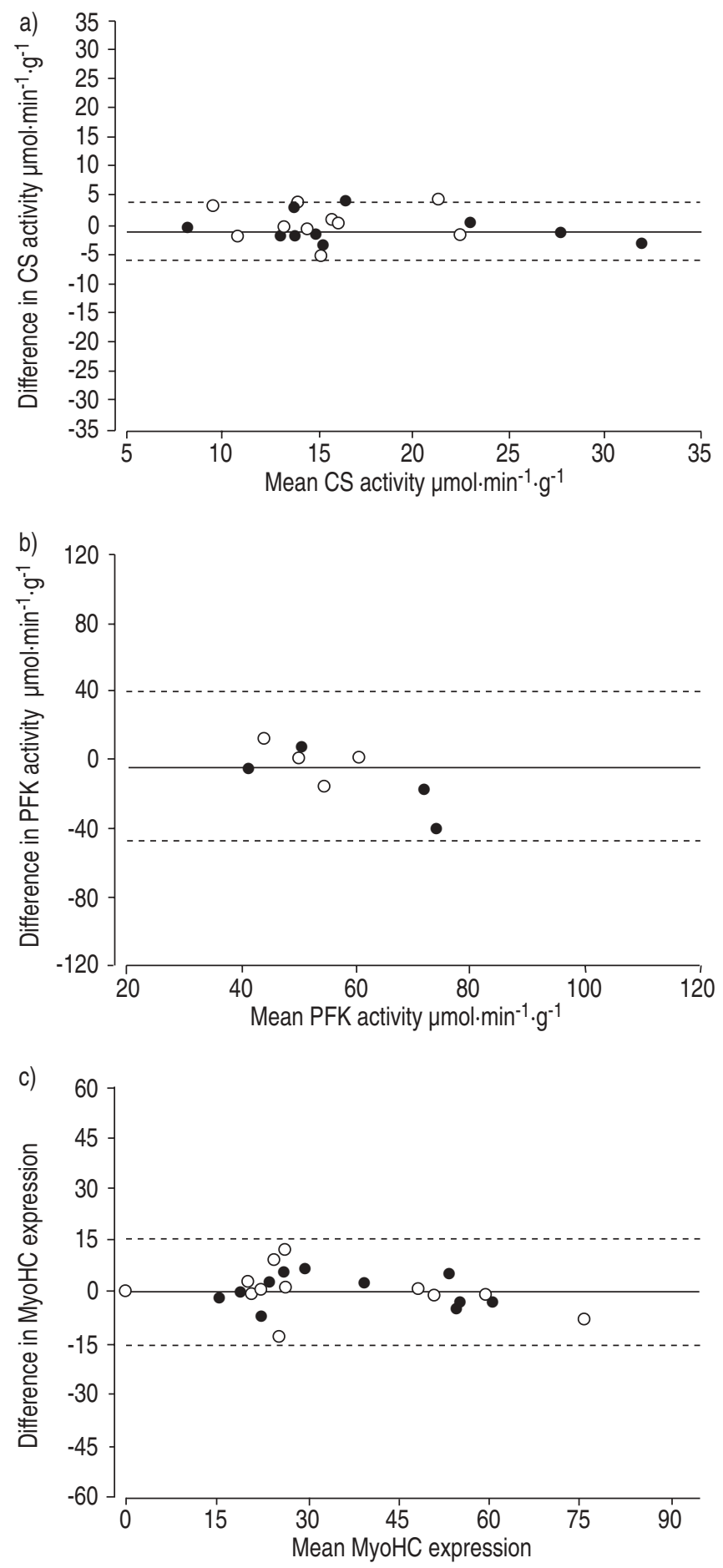

FIGURE 5. Illustration of the agreement between the two biopsy techniques in subjects in whom two pairs of biopsies were obtained, according to the Bland and Altman graphical representation. a) Individual results of citrate synthase (CS) activity in 10 subjects ( $\bullet$ : first biopsy; $\bigcirc$ : second biopsy). The mean difference $(-)$ and the limits of agreement (----) are those of the whole group, i.e. as in figure 2b. b) Individual results of phosphofructokinase activity (PFK) in four subjects. The mean difference and the limits of agreement are those of the whole group, i.e. as in figure 3b. c) Bland and Altman graphical representation of myosin heavy chain (MyoHC) expression where the different isoforms of four subjects were pooled. The mean difference and the limits of agreement are the same as in figure $4 \mathrm{~b}$. 

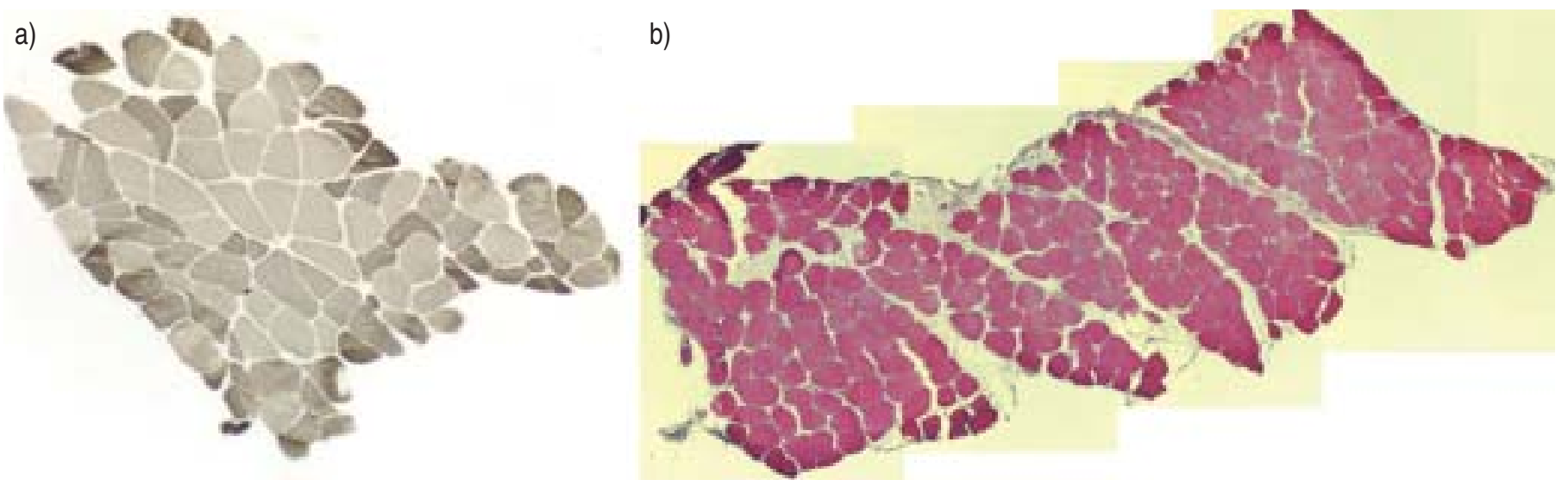

FIGURE 6. Examples of muscle transversal sections obtained with the microbiopsy procedure at the beginning (a) and by the end (b) of the trial.

the study sessions with better tissue handling and freezing procedures. Figure 6 shows a transversal section from a microbiopsy sample taken at the beginning of the study, which was nevertheless of good quality (fig. 6a), and from a sample taken at the end (fig. $6 \mathrm{~b}$ ). The number of muscle fibres could be determined in 10 out of the 12 microbiopsy specimens studied. The median (range) cell number was 144 (38-286).

\section{DISCUSSION}

The present study shows the feasibility of using a minimally invasive technique to biopsy the vastus lateralis in COPD patients and healthy volunteers. In addition, when compared with the Bergström biopsy technique, the current gold standard, the microbiopsy provided excellent agreement for $\mathrm{CS}$ and MyoHC composition. For PFK, the agreement was only moderate, perhaps because of greater variability in the expression of this enzyme in human skeletal muscle. The agreement between the two biopsy techniques persisted when two separate biopsies of each type were obtained from the same subjects. Some of the microbiopsy specimens were also useful for microscopic analyses. Thus, microbiopsy appears to be a valid technique for studying the peripheral muscles of COPD patients.

\section{Skeletal muscle biopsy techniques and tissue sample quality}

A percutaneous microbiopsy was used with the aim of reducing the needle size to improve patient acceptance and tolerance, while ensuring a sufficient muscle sample size at the same time. The technique most often used to assess the vastus lateralis muscle in patients with COPD or other chronic diseases is the Bergström semi-open biopsy, which is generally performed with $6-\mathrm{mm}$ trocars and requires a $0.5-1-\mathrm{cm}$ skin incision. Open biopsies are also feasible in these patients [6, 7], but are even more invasive than the Bergström technique. Both approaches have the advantage of providing abundant muscle tissue for reliable biochemical, histochemical and histomorphometric analyses [24], but their major drawback is limited patient acceptance and the difficulty of obtaining serial biopsies in a given subject. Using a 17-gauge needle and a different automated device than that of the current authors, VEscovo et al. [13, 14] showed that it is possible to determine the MyoHC composition of the gastrocnemius muscle in patients with chronic heart failure with the microbiopsy procedure. However, their specimen weighed only 50$200 \mu \mathrm{g}$, which is too little for enzymatic activity or histological studies. Using a 16-gauge needle and the Magnum ${ }^{\circledR}$ Biopsy System (Bard), muscle specimens of sufficient mean total weight (from $15 \mathrm{mg}$ at the beginning of the study to $55 \mathrm{mg}$ by the end) were obtained, while still ensuring patient comfort. Although there was a learning process with the use of the microbiopsy, the amount of muscle tissue could be easily increased with some experience up to $60-90 \mathrm{mg}$. This might be sufficient to perform the biochemical and cellular analyses required for several purposes in COPD patients [25] and patients with neuromuscular disorders [12]. In addition, nutritional status did not appear to be a limitation to the microbiopsy technique.

Some of the sections from the microbiopsy specimens were observed under light microscopy and appeared to be of good quality, with a median of 144 cells counted on a single section. The current results were lower than those in two previous reports using similar automated devices. COTÉ et al. [11] reported fibre counts ranging 200-500 per section, and MAGISTRIs et al. [12] were able to reach up to 1,000 muscle fibres in their biopsy specimens. However, it should be emphasised that, in both studies, larger needles than those from the current study were used (14 or 15-gauge). Since a precise histological diagnosis was essential in their patients, these authors used a stereomicroscope to ensure good sample orientation, and were assisted by an experienced pathologist. In the current study, these precautions would probably have improved the quality of the microbiopsy sections and increased the number of cells counted. However, as can be seen in figure 6, substantial improvement was gradually obtained in the tissue quality over the course of the study as the current authors gained experience with the technique. Therefore, the present results are encouraging. Now that the feasibility and validity of the microbiopsy technique have been confirmed, further work will be required to establish the usefulness of the microbiopsy approach for cytological, histomorphometric and immunostaining analyses. However, other investigators have reported interesting data on the applicability of various cellular techniques on small biopsy samples [12]. Therefore, the current authors' method is also likely to be useful for these other biological techniques. 


\section{Clinical tolerance}

The microbiopsy was very well tolerated, since subjects reported little or no pain (much less than with the Bergström biopsy) and all said they would definitely prefer the microbiopsy in the future. As the microbiopsy was immediately followed by the Bergström biopsy, the time-course of any lateoccurring discomfort due to the microbiopsy could not be evaluated. Using 14-gauge needles alone in 98 adults with suspicion of neuromuscular disease, COTÉ et al. [11] reported one case of a peculiar pain syndrome that lasted several weeks. With similar needles, MAGISTRIS et al. [12] reported a punching sensation in some patients lasting up to 4 days.

In the current study, one or two passes were made with the Bergström needle, which is the present authors' usual practice and sufficient to obtain enough tissue. For the microbiopsy, an original procedure was developed, which has not been used in earlier fine-needle biopsy studies [10-12]. The major modification was the use of an insertion cannula (a 14-gauge i.v. catheter) to obtain several biopsy samples through a single skin puncture, thus obviating the need for multiple punctures. When the microbiopsy needle was slid out of the cannula, the latter was maintained in place without any pain or discomfort to the subjects. This system avoids repeated skin punctures when several samplings are needed, because it is easy to reintroduce the microbiopsy needle directly into the biopsy site without contact with the incision or the muscle fascia. This is in contrast with the Bergström method, for which the entire needle needs to be removed and then reintroduced through the skin incision when a second sample is needed, a procedure that is often described as causing an uncomfortable sensation. Another difference between the two methods is the need to create suction for the Bergström biopsy in order to capture and cut the muscle sample [4]. This is generally the most painful part of the Bergström technique. In contrast, the microbiopsy technique does not require suction and, therefore, even when six samplings are made, the subject is generally perfectly relaxed. In the present study, the subjects' unanimous choice of the microbiopsy technique for a further biopsy leaves no doubt as to the greater comfort of this technique.

Haematoma is the most often reported complication after muscle biopsy, but it remains infrequent. MAGISTRIS et al. [12] only observed two haematomas out of 220 fine-needle biopsies using a larger size than in the current study, with no sequelae. By avoiding microbiopsies in patients with anticoagulation or blood discrasia, the risk of complication with microbiopsy can be reduced even more. In summary, microbiopsy can be considered to be a very safe procedure.

Another advantage of the microbiopsy procedure is that it can be performed without skin incision, thus facilitating patient acceptance of the procedure, particularly when repeated biopsies are needed. Thus, the microbiopsy technique reported here may have both ethical and cosmetic arguments in its favour.

\section{Biological comparisons between microbiopsy and the Bergström technique}

The enzymatic activities of CS and PFK were determined with 5-10-mg samples from the microbiopsy and the Bergström biopsy, with excellent agreement for the duplicate measurements of both enzymes (data not shown), in line with previous reports underlining the high level of reproducibility of repeated assays from the same homogenate [24].

Excellent agreement was found between the two biopsy techniques for CS activity and MyoHC composition, but only moderate agreement for PFK. All together, the current results are in accordance with those of SIMONEAU et al. [24], who found no significant change in the fibre-type distribution or the mean activities of several enzymes obtained from two separate biopsies in the same vastus lateralis of normal subjects, and when right and left sides were compared in the same subjects. However, as was observed in the present study, SIMONEAU et al. [24] found intra-subject typological or enzymatic activity variability, particularly for PFK activity, for which the coefficient of correlation ranged from 0.71 for comparison of the same muscle to 0.63 for comparisons of the two sides. This variability of PFK was attributed to possible differences in the depth of muscle sampling, since variation in biochemical results may exist in muscle samples taken $1 \mathrm{~cm}$ apart [24]. In this study, the current authors tried to perform the microbiopsy and the Bergström biopsy at the same site, but it is likely that the two samples came from slightly different muscle areas. Variability may also have been due to differences in the composition of the homogenates, in terms of muscle or nonmuscle tissue, or to differences in laboratory technical procedures [24]. In this regard, care was taken to minimise technical errors by measuring the muscle enzyme activity of the two biopsy samples from each subject simultaneously. The possibility that some of the microbiopsy or Bergström biopsy specimens contained small amounts of fat tissue (perhaps from subcutaneous tissue), which may express greater PFK than CS activity, cannot be excluded.

The present study showed that the agreement between the two biopsy techniques persisted in subjects in whom two biopsies of each type were obtained, even though the experimental conditions were different. Since the two pairs of biopsies were not obtained under the same conditions, the repeatability of the microbiopsy could not be directly evaluated. Previous studies have shown 15-25\% variation in enzyme activities in repeated Bergström biopsies of the vastus lateralis muscle [24], and it is unlikely that the repeatability of the microbiopsy would be better. However, the current observations argue for a repeatability of the microbiopsy that is certainly comparable with that of the Bergström technique, not only over time, but also over different experimental conditions. In this regard, the use of microbiopsies could be envisaged to evaluate with confidence, for example, the effects of several interventions after which biological values are assumed to vary, such as pulmonary rehabilitation [9].

\section{Perspectives for the microbiopsy}

At the beginning of the study, the present authors aimed to reconcile two different requirements for clinical research on the skeletal muscle dysfunction of COPD patients. The first inevitable requirement is the muscle biopsy, since research at the cellular level is essential [9, 26-28]. The second requirement, however, is to pursue research goals as noninvasively as possible in these patients [29], which compelled the current authors to search for an acceptable alternative to the Bergström 
technique. It is believed that the present study presents a promising tool to reliably and noninvasively assess the skeletal muscle in COPD patients. However, the potential applications of the current authors' technique are probably broader than their initial aim. It should now be easier to repeat microbiopsies in the same subject with little discomfort, in order to study, for example, the responses over time of various metabolic pathways to a therapeutic intervention. As the technique requires limited equipment, is easy to learn, and has a low rate of complication, the microbiopsy could be used for a much wider range of outpatients. This would facilitate multicentric studies with large populations of patients who could be assessed at the muscle level. Another advantage is that several other peripheral muscles can be biopsied with this technique [12], so several sites in the same patient could be sampled with minimal discomfort. Finally, this technique may also be of great interest for numerous chronic diseases or for physiological research in normal subjects.

In conclusion, this study shows the feasibility and validity of a minimally invasive technique for the biopsy of the vastus lateralis muscle: the microbiopsy. Compared with the current gold standard, the Bergström technique, the microbiopsy provides excellent results with regards to citrate synthase activity and to the distribution of myosin heavy chain composition. Numerous applications of this technique can be envisioned, notably in the investigation of peripheral muscle dysfunction and its underlying mechanisms, not only in chronic obstructive pulmonary disease patients, but also in patients with numerous other chronic diseases.

\section{ACKNOWLEDGEMENTS}

The authors are grateful to M. Bélanger, M-J. Breton and B. Jean for their enthusiastic assistance, and to J-P. Meullenet for his collaboration.

\section{REFERENCES}

1 Skeletal muscle dysfunction in chronic obstructive pulmonary disease. A statement of the American Thoracic Society and European Respiratory Society. Am J Respir Crit Care Med 1999; 159: S1-S40.

2 Agusti AG, Noguera A, Sauleda J, Sala E, Pons J, Busquets $X$. Systemic effects of chronic obstructive pulmonary disease. Eur Respir J 2003; 21: 347-360.

3 Bergström J. Muscle electrolytes in man. Determination by neutron activation analysis on needle biopsy specimens. A study on normal subjects, kidney patients and patients with chronic diarrhoea. Scand J Clin Lab Invest 1962; 14 : $1-110$.

4 Hennessey JV, Chromiak JA, Della Ventura S, Guertin J, MacLean DB. Increase in percutaneous muscle biopsy yield with a suction-enhancement technique. J Appl Physiol 1997; 82: 1739-1742.

5 Gosker HR, Kubat B, Schaart G, van der Vusse GJ, Wouters EF, Schols AM. Myopathological features in skeletal muscle of patients with chronic obstructive pulmonary disease. Eur Respir J 2003; 22: 280-285.

6 Debigare R, Cote $\mathrm{CH}$, Hould FS, LeBlanc P, Maltais F. In vitro and in vivo contractile properties of the vastus lateralis muscle in males with COPD. Eur Respir J 2003; 21: 273-278.
7 Barreiro E, Gea J, Corominas JM, Hussain SN. Nitric oxide synthases and protein oxidation in the quadriceps femoris of patients with chronic obstructive pulmonary disease. Am J Respir Cell Mol Biol 2003; 29: 771-778.

8 Gea JG, Pasto M, Carmona MA, Orozco-Levi M, Palomeque J, Broquetas J. Metabolic characteristics of the deltoid muscle in patients with chronic obstructive pulmonary disease. Eur Respir J 2001; 17: 939-945.

9 Maltais F, LeBlanc P, Simard C, et al. Skeletal muscle adaptation to endurance training in patients with chronic obstructive pulmonary disease. Am J Respir Crit Care Med 1996; 154: 442-447.

10 Welker JA, Henshaw RM, Jelinek J, Shmookler BM, Malawer MM. The percutaneous needle biopsy is safe and recommended in the diagnosis of musculoskeletal masses. Cancer 2000; 89: 2677-2686.

11 Cote AM, Jimenez L, Adelman LS, Munsat TL. Needle muscle biopsy with the automatic Biopty instrument. Neurology 1992; 42: 2212-2213.

12 Magistris MR, Kohler A, Pizzolato G, et al. Needle muscle biopsy in the investigation of neuromuscular disorders. Muscle Nerve 1998; 21: 194-200.

13 Vescovo G, Dalla Libera L, Serafini F, et al. Improved exercise tolerance after losartan and enalapril in heart failure: correlation with changes in skeletal muscle myosin heavy chain composition. Circulation 1998; 98: 1742-1749.

14 Vescovo G, Serafini F, Facchin L, et al. Specific changes in skeletal muscle myosin heavy chain composition in cardiac failure: differences compared with disuse atrophy as assessed on microbiopsies by high resolution electrophoresis. Heart 1996; 76: 337-343.

15 Pauwels RA, Buist AS, Calverley PM, Jenkins CR, Hurd SS. Global strategy for the diagnosis, management, and prevention of chronic obstructive pulmonary disease. NHLBI/WHO Global Initiative for Chronic Obstructive Lung Disease (GOLD) Workshop summary. Am J Respir Crit Care Med 2001; 163: 1256-1276.

16 Hopper KD, Abendroth CS, Sturtz KW, Matthews YL, Stevens LA, Shirk SJ. Automated biopsy devices: a blinded evaluation. Radiology 1993; 187: 653-660.

17 Borg G. Psychological bases of perceived exertion. Med Sci Sports Exerc 1982; 14: 377-381.

18 Zammit VA, Newsholme EA. The maximum activities of hexokinase, phosphorylase, phosphofructokinase, glycerol phosphate dehydrogenases, lactate dehydrogenase, octopine dehydrogenase, phosphoenolpyruvate carboxykinase nucleoside diphosphatekinase, glutamate-oxaloacetate transaminase and arginine kinase in relation to carbohydrate utilization in muscles from marine invertebrates. Biochem J 1976; 160: 447-462.

19 Srere PA. Citrate synthase. Methods Enzymol 1969; 13: 3-11.

20 Bradford MM. A rapid and sensitive method for the quantitation of microgram quantities of protein utilizing the principle of protein-dye binding. Anal Biochem 1976; 72: 248-254

21 Ennion S, Sant'ana Pereira J, Sargeant AJ, Young A, Goldspink G. Characterization of human skeletal muscle fibres according to the myosin heavy chains they express. J Muscle Res Cell Motil 1995; 16: 35-43. 
22 Smerdu V, Karsch-Mizrachi I, Campione M, Leinwand L, Schiaffino S. Type IIx myosin heavy chain transcripts are expressed in type IIb fibers of human skeletal muscle. Am J Physiol 1994; 267: C1723-C1728.

23 Bland JM, Altman DG. Statistical methods for assessing agreement between two methods of clinical measurement. Lancet 1986; 1: 307-310.

24 Simoneau JA, Lortie G, Boulay MR, Thibault MC, Bouchard C. Repeatability of fibre type and enzyme activity measurements in human skeletal muscle. Clin Physiol 1986; 6: 347-356.

25 Pouw EM, Koerts-de Lang E, Gosker HR, et al. Muscle metabolic status in patients with severe COPD with and without long-term prednisolone. Eur Respir J 2000; 16: 247-252.
26 Couillard A, Maltais F, Saey D, et al. Exercise-induced quadriceps oxidative stress and peripheral muscle dysfunction in patients with chronic obstructive pulmonary disease. Am J Respir Crit Care Med 2003; 167: 16641669.

27 Maltais F, LeBlanc P, Whittom F, et al. Oxidative enzyme activities of the vastus lateralis muscle and the functional status in patients with COPD. Thorax 2000; 55: 848-853.

28 Maltais F, Sullivan MJ, LeBlanc P, et al. Altered expression of myosin heavy chain in the vastus lateralis muscle in patients with COPD. Eur Respir J 1999; 13: 850-854.

29 Hayot M, Ramonatxo M, Matecki S, Milic-Emili J, Prefaut C. Noninvasive assessment of inspiratory muscle function during exercise. Am J Respir Crit Care Med 2000; 162: 2201-2207. 